\title{
The distribution of ascents of size $d$ or more in samples of geometric random variables
}

\author{
Charlotte Brennan ${ }^{1}$ and Arnold Knopfmacher ${ }^{1 \dagger}$ \\ ${ }^{1}$ The John Knopfmacher Centre for Applicable Analysis and Number Theory, School of Mathematics, \\ University of the Witwatersrand, Private Bag 3, Johannesburg, South Africa.
}

We consider words or strings of characters $a_{1} a_{2} a_{3} \ldots a_{n}$ of length $n$, where the letters $a_{i} \in \mathbb{N}$ are independently generated with a geometric probability

$$
\mathbb{P}\{X=k\}=p q^{k-1} \text { where } p+q=1 .
$$

Let $d$ be a fixed nonnegative integer. We say that we have an ascent of size $d$ or more if $a_{i+1} \geq a_{i}+d$. We determine the mean, variance and limiting distribution of the number of ascents of size $d$ or more in a random geometrically distributed word.

Keywords: geometric random variables, distributions, generating functions

\section{Introduction}

Suppose we have words or strings of characters from a fixed alphabet, $a_{1} a_{2} a_{3} \ldots a_{n}$ of length $n$, where the letters $a_{i} \in \mathbb{N}$. These letters are independently generated with a geometric probability such that

$$
\mathbb{P}\{X=k\}=p q^{k-1} \text { where } p+q=1
$$

Let $d$ be a fixed nonnegative integer. We say that we have an ascent of size $d$ or more (called a $d$-ascent) if $a_{i+1} \geq a_{i}+d$. In Section 2 we find the average number of ascents of size $d$ or more in a sample of geometric random variables. In subsequent sections we determine the associated variance and distribution. Recently in [6], weak and strict descents in samples of geometric random variables were studied, which is equivalent to the special cases $d=0$ and $d=1$ of our results. However, the approach used in [6] does not generalize to ascents with $d \geq 2$.

Note that from a probabilistic perspective the central limit theorem for $d$-ascents holds also for samples generated by more general distributions than just the geometric one. Any distribution with bounded second moment could be used: Denote an ascent of size $d$ or more from $a_{i}$ to $a_{i+1}$ by the indicator variable $Y_{i}$. Then the total number of $d$-ascents is a sum of 2-dependent random variables (i.e. $Y_{n}$ and $Y_{m}$ are independent for $|n-m| \geq 2$ ). General probability theory (see for example [2], Section 27) then implies a linear mean and variance as well as the central limit law.

The purpose of this paper is to give an alternate approach in the particular case of geometric random variables in which it is possible to obtain much more precise results than in general, such as an explicit bivariate

${ }^{\dagger}$ This material is based upon work supported by the National Research Foundation under grant number 2053740 
generating function and explicit mean and variance. In addition, with respect to the central limit theorem, our explicit approach means that it would be possible in theory to compute a full asymptotic series expansion of the error term.

\section{Expected number of ascents of size $d$ or more in samples of geo- metric random variables}

\subsection{Using a probability generating function}

We use the "adding the slice" technique which was originally used by P. Flajolet and H. Prodinger in [3] and more recently by A. Knopfmacher and H. Prodinger in [5].

Let $j$ be the value of the last component of the word, i.e., $a_{k}=j$. We proceed from a sample with $k$ parts to a sample with $k+1$ parts. We denote by $f_{k}(z, u, v)$ the generating function where $z$ counts the length $k$ of the word, $u$ the value of $j$ and $v$ counts the number of ascents of size $d$ or more. That is, $\left[z^{k} u^{j} v^{l}\right] f_{k}(z, u, v)$ gives the probability that a geometric word of length $k$ has last component equal to $j$ and $l$ ascents of size $d$ or more, and $\left[z^{m} u^{j} v^{l}\right] f_{k}(z, u, v)=0$ for $m \neq k$.

In moving from a sample with $k$ parts to a sample of $k+1$ parts, where $j$ is coded by $u^{j}$, we have an ascent of size $d$ or more, coded by $v$, whenever the new last letter has any value from $j+d$ onwards. This gives the following rule for adding a new part ("slice") to the end of a word:

$$
\begin{aligned}
& u^{j} \longrightarrow z p u+z p q u^{2}+\cdots+z p q^{j+d-2} u^{j+d-1}+v z\left\{p q^{j+d-1} u^{j+d}+p q^{j+d} u^{j+d+1}+\cdots\right\} \\
& =p z u \frac{1-(q u)^{j+d-1}}{1-q u}+v z p q^{j+d-1} u^{j+d} \frac{1}{1-q u} .
\end{aligned}
$$

This implies that

$$
\begin{aligned}
f_{k+1}(z, u, v) & =\frac{p z u}{1-q u} f_{k}(z, 1, v)-\frac{p z u(q u)^{d-1}}{1-q u} f_{k}(z, q u, v)+\frac{v p z u^{d} q^{d-1}}{1-q u} f_{k}(z, q u, v) \\
& =\frac{p z u}{1-q u} f_{k}(z, 1, v)-\frac{(1-v) p q^{d-1} z u^{d}}{1-q u} f_{k}(z, q u, v) .
\end{aligned}
$$

Now define

$$
F(z, u, v):=\sum_{k \geq 1} f_{k}(z, u, v)
$$

Summing (2.1) over $k \geq 1$ :

$$
F(z, u, v)-f_{1}(z, u, v)=\frac{p z u}{1-q u} F(z, 1, v)-\frac{(1-v) p q^{d-1} z u^{d}}{1-q u} F(z, q u, v),
$$

so that

$$
F(z, u, v)=\frac{p z u}{1-q u} F(z, 1, v)+\frac{p z u}{1-q u}-\frac{(1-v) p q^{d-1} z u^{d}}{1-q u} F(z, q u, v),
$$

where we have used

$$
f_{1}(z, u, v)=z p u+z p q u^{2}+z p q^{2} u^{3}+\cdots=\frac{p z u}{1-q u} .
$$


At this stage we iterate the recursion for $F(z, u, v)$.

$$
\begin{aligned}
& F(z, u, v)=\frac{p z u}{1-q u} F(z, 1, v)+\frac{p z u}{1-q u}-\frac{(1-v) p q^{d-1} z u^{d}}{1-q u} \times \\
& \quad \times\left\{\frac{p q z u}{1-q^{2} u} F(z, 1, v)+\frac{p q z u}{1-q^{2} u}-\frac{(1-v) p q^{d-1} z(q u)^{d}}{1-q^{2} u} F\left(z, q^{2} u, v\right)\right\} \\
& =\left[\frac{p z u}{1-q u}-\frac{(1-v) p q^{d-1} z u^{d} p q z u}{(1-q u)\left(1-q^{2} u\right)}\right][F(z, 1, v)+1]+\frac{(1-v)^{2}\left(p q^{d-1} z\right)^{2} u^{d}(q u)^{d}}{(1-q u)\left(1-q^{2} u\right)} \times \\
& \quad \times\left\{\frac{p q^{2} z u}{1-q^{3} u} F(z, 1, v)+\frac{p q^{2} z u}{1-q^{3} u}-\frac{(1-v) p q^{d-1} z\left(q^{2} u\right)^{d}}{1-q^{3} u} F\left(z, q^{3} u, v\right)\right\} \\
& =\left[\frac{p z u}{1-q u}-\frac{(1-v) p q^{d-1} z u^{d} p q z u}{(1-q u)\left(1-q^{2} u\right)}+\frac{(1-v)^{2}\left(p q^{d-1} z\right)^{2} u^{d} p(q u)^{d} p q^{2} z u}{(1-q u)\left(1-q^{2} u\right)\left(1-q^{3} u\right)}\right][F(z, 1, v)+1] \\
& \quad-\frac{(1-v)^{3}\left(p q^{d-1} z\right)^{3} u^{d}(q u)^{d}\left(q^{2} u\right)^{d}}{(1-q u)\left(1-q^{2} u\right)\left(1-q^{3} u\right)} F\left(z, q^{3} u, v\right) .
\end{aligned}
$$

We keep iterating and put $u=1$ to obtain

$$
\begin{aligned}
F(z, 1, v)= & {\left[\frac{p z}{1-q}-\frac{(1-v) p q^{d-1} z p q z}{(1-q)\left(1-q^{2}\right)}+\frac{(1-v)^{2}\left(p q^{d-1} z\right)^{2} q^{d} p q^{2} z}{(1-q)\left(1-q^{2}\right)\left(1-q^{3}\right)}\right.} \\
& \left.-\frac{(1-v)^{3}\left(p q^{d-1} z\right)^{3} q^{d} q^{2 d} p q^{3} z}{(1-q)\left(1-q^{2}\right)\left(1-q^{3}\right)\left(1-q^{4}\right)}+\cdots\right][F(z, 1, v)+1] \\
= & \sum_{i \geq 1} \frac{(1-v)^{i-1}(-1)^{i-1} p^{i} q^{\frac{d i(i-1)}{2}} z^{i}}{(1-q)\left(1-q^{2}\right) \cdots\left(1-q^{i}\right)}[F(z, 1, v)+1] .
\end{aligned}
$$

Therefore we find

\section{Proposition 1}

$$
F(z, 1, v)=\frac{\sigma(z, v)}{1-\sigma(z, v)}
$$

where

$$
\sigma(z, v):=\sum_{i \geq 1} \frac{(1-v)^{i-1}(-1)^{i-1} p^{i} q^{d\left(\begin{array}{c}
i \\
2
\end{array}\right)} z^{i}}{(1-q)\left(1-q^{2}\right) \cdots\left(1-q^{i}\right)} .
$$

The expected value is $\left.\left[z^{n}\right] \frac{\partial F}{\partial v}\right|_{v=1}$. For this we shall need

$$
\left.\sigma(z, v)\right|_{v=1}=\frac{p z}{1-q}=z
$$

and

$$
\left.\sigma^{\prime}(z, v)\right|_{v=1}=\left.\sum_{i \geq 1} \frac{(i-1)(1-v)^{i-2}(-1)^{i} p^{i} q^{d\left(\begin{array}{c}
i \\
2
\end{array}\right)} z^{i}}{(1-q)\left(1-q^{2}\right) \cdots\left(1-q^{i}\right)}\right|_{v=1}=\frac{q^{d} z^{2}}{1+q}
$$

Hence

$$
\left.\frac{\partial F}{\partial v}\right|_{v=1}=\left.\frac{\sigma^{\prime}(z, v)}{(1-\sigma(z, v))^{2}}\right|_{v=1}=\frac{q^{d} z^{2}}{(1+q)(1-z)^{2}} .
$$


So that

$$
\mathbb{E}(n)=\left.\left[z^{n}\right] \frac{\partial F}{\partial v}\right|_{v=1}=\left[z^{n-2}\right] \frac{q^{d}}{(1+q)(1-z)^{2}}=(n-1) \frac{q^{d}}{1+q} .
$$

Thus we have shown

Theorem 1 The expected number of ascents of size d or more in a word consisting of $n$ geometric random variables is

$$
\mathbb{E}(n)=(n-1) \frac{q^{d}}{1+q} .
$$

For the special cases $d=0$ and $d=1$, these results can be found in [6], which studies descents in a sample of geometric random variables. The number of weak descents, where $a_{i} \geq a_{i+1}$, corresponds by reversing the string of characters, to our ascents of size 0 or more. As for $d=1$, it is the same by reversing words, as the number of strict descents, where $a_{i}>a_{i+1}$.

\subsection{Using discrete probability}

In the case of the mean, a shorter approach is available. In a word of length $n$, there are $n-1$ adjacent pairs of characters. We can use the additive property of the means to calculate the average number of ascents of size $d$ or more per word of length $n$, just by calculating the probability of having an ascent of size $d$ or more in a pair say $a_{k}$ to $a_{k+1}$ and then multiply by $n-1$. We need $a_{i+1} \geq a_{i}+d$. For each pair this will occur with probability:

$$
\sum_{i=1}^{\infty} p q^{i-1} \sum_{j=d+i}^{\infty} p q^{j-1}=\frac{q^{d}}{1+q} .
$$

\subsection{Samples of geometric random variables having no ascents of size $d$ or more}

Let $F_{d}(z)$ be the generating function for samples of geometric random variables having no ascents of size $d$ or more. For convenience the generating function will include a term 1 for the empty word. Then using (2.2) and (2.3),

$$
F_{d}(z)=1+F(z, 1,0)=\frac{1}{1-\sigma_{d}(z, 1)},
$$

where

$$
\sigma_{d}(z, 1):=\sum_{i \geq 1} \frac{(-1)^{i-1} p^{i} q^{d\left(\begin{array}{c}
i \\
2
\end{array}\right)} z^{i}}{(1-q)\left(1-q^{2}\right) \cdots\left(1-q^{i}\right)} .
$$

For a few special choices of $d$ we can make use of partition identities to rewrite $F_{d}(z)$ in the form of an infinite product. By using Euler's partition identities (see [1])

$$
\sum_{n=0}^{\infty} \frac{x^{n}}{(1-q)\left(1-q^{2}\right) \cdots\left(1-q^{n}\right)}=\prod_{i \geq 0} \frac{1}{1-x q^{i}}
$$

and

$$
\sum_{n=0}^{\infty} \frac{(-1)^{n} x^{n} q^{\left(\begin{array}{c}
n \\
2
\end{array}\right)}}{(1-q)\left(1-q^{2}\right) \cdots\left(1-q^{n}\right)}=\prod_{i \geq 0}\left(1-x q^{i}\right)
$$


we find that

$$
F_{0}(z)=\prod_{i \geq 0}\left(1+p z q^{i}\right)
$$

and

$$
F_{1}(z)=\prod_{i \geq 0} \frac{1}{1-p z q^{i}} .
$$

These formulas have a natural interpretation as the generating functions of strictly ascending geometric words and of weakly ascending geometric words, respectively. There do not appear to be analogous product expressions for $d \geq 2$. However, for $d=2$ we can make use of the Rogers-Ramanujan identities ( [1])

$$
\sum_{n=0}^{\infty} \frac{q^{n^{2}+n}}{(1-q)\left(1-q^{2}\right) \cdots\left(1-q^{n}\right)}=\prod_{i \geq 0} \frac{1}{\left(1-q^{5 i+2}\right)\left(1-q^{5 i+3}\right)}
$$

and

$$
\sum_{n=0}^{\infty} \frac{q^{n^{2}}}{(1-q)\left(1-q^{2}\right) \cdots\left(1-q^{n}\right)}=\prod_{i \geq 0} \frac{1}{\left(1-q^{5 i+1}\right)\left(1-q^{5 i+4}\right)}
$$

to obtain for the values $z=-q^{2} / p$ and $z=-q / p$, the curious infinite product formulas

$$
F_{2}\left(-\frac{q^{2}}{p}\right)=\prod_{i \geq 0}\left(1-q^{5 i+2}\right)\left(1-q^{5 i+3}\right)
$$

and

$$
F_{2}\left(-\frac{q}{p}\right)=\prod_{i \geq 0}\left(1-q^{5 i+1}\right)\left(1-q^{5 i+4}\right) .
$$

\section{Variance of the number of ascents of size $d$ or more in samples of geometric random variables}

Recall the following results from Section 2:

$$
F(z, 1, v)=\frac{\sigma(z, v)}{1-\sigma(z, v)},
$$

where

In addition

$$
\begin{gathered}
\sigma(z, v)=\sum_{i \geq 1} \frac{(1-v)^{i-1}(-1)^{i-1} p^{i} q^{d\left(\begin{array}{c}
i \\
2
\end{array}\right)} z^{i}}{(1-q)\left(1-q^{2}\right) \cdots\left(1-q^{i}\right)} \\
\left.\sigma(z, v)\right|_{v=1}=z \\
\left.\sigma^{\prime}(z, v)\right|_{v=1}=\frac{q^{d} z^{2}}{1+q}
\end{gathered}
$$

$$
\begin{aligned}
\left.\sigma^{\prime \prime}(z, v)\right|_{v=1} & =\left.\sum_{i \geq 1} \frac{(i-2)(i-1)(1-v)^{i-3}(-1)^{i-1} p^{i} q^{d\left(\begin{array}{c}
i \\
2
\end{array}\right)} z^{i}}{(1-q)\left(1-q^{2}\right) \cdots\left(1-q^{i}\right)}\right|_{v=1} \\
& =\frac{2 p q^{3 d} z^{3}}{(1+q)\left(1-q^{3}\right)}
\end{aligned}
$$


Therefore

$$
\begin{aligned}
\left.\frac{\partial^{2} F}{\partial v^{2}}\right|_{v=1} & =\left.\frac{(1-\sigma) \sigma^{\prime \prime}+2 \sigma^{\prime 2}}{(1-\sigma)^{3}}\right|_{v=1} \\
& =\frac{2 p q^{3 d} z^{3}}{(1+q)\left(1-q^{3}\right)(1-z)^{2}}+\frac{2 q^{2 d} z^{4}}{(1+q)^{2}(1-z)^{3}}
\end{aligned}
$$

which means that

$$
\begin{aligned}
{\left.\left[z^{n}\right] \frac{\partial^{2} F}{\partial v^{2}}\right|_{v=1} } & =\left[z^{n-3}\right] \frac{2 p q^{3 d}}{(1+q)\left(1-q^{3}\right)(1-z)^{2}}+\left[z^{n-4}\right] \frac{2 q^{2 d}}{(1+q)^{2}(1-z)^{3}} \\
& =(n-2) \frac{2 p q^{3 d}}{(1+q)\left(1-q^{3}\right)}+(n-3)(n-2) \frac{q^{2 d}}{(1+q)^{2}} .
\end{aligned}
$$

So finally, after adding the expectation and subtracting the square of the expectation we obtain the variance

$$
\begin{aligned}
\mathbb{V}(n) & =(n-2) \frac{2 p q^{3 d}}{(1+q)\left(1-q^{3}\right)}+(n-3)(n-2) \frac{q^{2 d}}{(1+q)^{2}}+(n-1) \frac{q^{d}}{1+q}-(n-1)^{2} \frac{q^{2 d}}{(1+q)^{2}} \\
& =(n-1) \frac{q^{d}\left(1+q-q^{d}\right)}{(1+q)^{2}}+2(n-2)\left(\frac{q^{3 d}(1-q)}{\left(1-q^{3}\right)(1+q)}-\frac{q^{2 d}}{(1+q)^{2}}\right) .
\end{aligned}
$$

Thus

Theorem 2 The variance of the number of ascents in samples of $n$ geometric random variables is

$$
\mathbb{V}(n)=\left[\frac{2 q^{3 d}(1-q)}{(1+q)\left(1-q^{3}\right)}-\frac{3 q^{2 d}}{(1+q)^{2}}+\frac{q^{d}}{1+q}\right] n-\frac{4 q^{3 d}(1-q)}{(1+q)\left(1-q^{3}\right)}+\frac{5 q^{2 d}}{(1+q)^{2}}-\frac{q^{d}}{1+q} .
$$

Remark It is also possible to find the variance by elementary statistical methods by computing the covariance between different pairs of adjacent random variables.

\section{Limiting distribution}

We are interested in finding the limiting distribution of our random variable. We make use of Proposition IX.8 from Flajolet and Sedgewick [4], which we state below for the convenience of the reader. We introduce the notation

$$
\mathfrak{v}(f)=\frac{f^{\prime \prime}(1)}{f(1)}+\frac{f^{\prime}(1)}{f(1)}-\left(\frac{f^{\prime}(1)}{f(1)}\right)^{2} .
$$

Proposition 2 "Meromorphic schema"

Let $F(z, u)$ be a bivariate function that is bivariate analytic at $(z, u)=(0,0)$ and has nonnegative coeffcients there. Assume that $F(z, 1)$ is meromorphic in $z \leq r$ with only a simple pole at $z=\rho$ for some positive $\rho<r$.

Assume also the following conditions.

i) Meromorphic pertubation: there exists $\epsilon>0$ and $r>\rho$ such that in the domain $\mathrm{D}=\{|z| \leq r\} \times\{\mid u-$ $1 \mid<\epsilon\}$, the function $F(z, u)$ admits the representation

$$
F(z, u)=\frac{B(z, u)}{C(z, u)},
$$


where $B(z, u), C(z, u)$ are analytic for $(z, u) \in D$ with $B(\rho, 1) \neq 0$. (Thus $\rho$ is a simple zero of $C(z, 1))$.

ii) Nondegeneracy: one has $\partial_{z} C(\rho, 1) \cdot \partial_{u} C(\rho, 1) \neq 0$, ensuring the existence of a nonconstant $\rho(u)$ analytic at $u=1$, such that $C(\rho(u), u)=0$ and $\rho(1)=\rho$.

iii) Variability: one has

$$
\mathfrak{v}\left(\frac{\rho}{\rho(u)}\right) \neq 0
$$

Then, the random variable with probability generating function

$$
p_{n}(u)=\frac{\left[z^{n}\right] F(z, u)}{\left[z^{n}\right] F(z, 1)}
$$

converges in distribution to a Gaussian variable with a speed of convergence that is $O\left(n^{-1 / 2}\right)$. The mean and the variance of $X_{n}$ are asymptotically linear in $n$.

In addition the following results are also given in Flajolet and Sedgewick [4]:

We introduce the notation

$$
c_{i, j}:=\left.\frac{\partial^{i+j}}{\partial z^{i} \partial u^{j}} C(z, u)\right|_{(\rho, 1)},
$$

then if $\rho(u)$ denotes the analytic solution of the implicit equation $C(\rho(u), u)=0$,

$$
\rho(u)=\rho-\frac{c_{0,1}}{c_{1,0}}(u-1)-\frac{c_{1,0}^{2} c_{0,2}-2 c_{1,0} c_{1,1} c_{0,1}+c_{2,0} c_{0,1}^{2}}{2 c_{1,0}^{3}}(u-1)^{2}+O\left((u-1)^{3}\right) .
$$

Condition (ii) corresponds to

$$
c_{0,1} c_{1,0} \neq 0 \text {. }
$$

The variability condition (iii) corresponds to

$$
\rho c_{1,0}^{2} c_{0,2}-\rho c_{1,0} c_{1,1} c_{0,1}+\rho c_{2,0} c_{0,1}^{2}+c_{0,1}^{2} c_{1,0}+c_{0,1} c_{1,0}^{2} \rho \neq 0 .
$$

For our specific problem

$$
F(z, 1, v)=\frac{\sigma(z, v)}{1-\sigma(z, v)} \equiv \frac{B(z, v)}{C(z, v)}
$$

so that

$$
C(z, v)=1-\sum_{i \geq 1} \frac{(1-v)^{i-1}(-1)^{i-1} p^{i} q^{d\left(\begin{array}{c}
i \\
2
\end{array}\right)} z^{i}}{(1-q)\left(1-q^{2}\right) \cdots\left(1-q^{i}\right)} .
$$

We have $\rho(1)=\rho=1$, since $1-\frac{p z}{1-q}=0$ when $z=1$.

We have according to (4.1)

$$
\begin{gathered}
c_{0,1}=\left.\frac{\partial}{\partial v} C(z, v)\right|_{(1,1)}=-\frac{q^{d}}{1+q} . \\
c_{1,0}=\left.\frac{\partial}{\partial z} C(z, v)\right|_{(1,1)}=-1 .
\end{gathered}
$$




$$
\begin{gathered}
c_{1,1}=\left.\frac{\partial^{2}}{\partial z \partial v} C(z, v)\right|_{(1,1)}=\frac{-2 q^{d}}{1+q} . \\
c_{0,2}=\left.\frac{\partial^{2}}{\partial v^{2}} C(z, v)\right|_{(1,1)}=\frac{-2 q^{3 d}}{(1+q)\left(1+q+q^{2}\right)} . \\
c_{2,0}=\left.\frac{\partial^{2}}{\partial z^{2}} C(z, v)\right|_{(1,1)}=0 .
\end{gathered}
$$

We are now in a position to check the conditions listed in the proposition.

For condition (ii), we need $c_{1,0} c_{0,1}=\frac{q^{d}}{1+q} \neq 0$, which is true for all $q>0$.

Using (4.2),

$$
\begin{aligned}
\rho(u) & =\rho-\frac{c_{0,1}}{c_{1,0}}(u-1)-\frac{c_{1,0}^{2} c_{0,2}-2 c_{1,0} c_{1,1} c_{0,1}+c_{2,0} c_{0,1}^{2}}{2 c_{1,0}^{3}}(u-1)^{2}+O\left((u-1)^{3}\right) \\
& =1-\frac{q^{d}}{1+q}(u-1)+\left(\frac{-q^{3 d}}{(1+q)\left(1+q+q^{2}\right)}+\frac{2 q^{2 d}}{(1+q)^{2}}\right)(u-1)^{2}+O\left((u-1)^{3}\right) .
\end{aligned}
$$

The variability condition (4.4) corresponds to computing

$$
\begin{aligned}
& \rho c_{1,0}^{2} c_{0,2}-\rho c_{1,0} c_{1,1} c_{0,1}+\rho c_{2,0} c_{0,1}^{2}+c_{0,1}^{2} c_{1,0}+c_{0,1} c_{1,0}^{2} \rho \\
& =\frac{-2 q^{3 d}}{(1+q)\left(1+q+q^{2}\right)}+\frac{2 q^{2 d}}{(1+q)^{2}}-\frac{q^{2 d}}{(1+q)^{2}}-\frac{q^{d}}{1+q} \\
& =\frac{-2 q^{3 d}}{(1+q)\left(1+q+q^{2}\right)}+\frac{q^{2 d}}{(1+q)^{2}}-\frac{q^{d}}{1+q} \\
& =-\frac{q^{d}\left(1+2 q+2 q^{2}+q^{3}-q^{d}-q^{d+1}-q^{d+2}+2 q^{2 d}+2 q^{2 d+1}\right)}{(1+q)^{2}\left(1+q+q^{2}\right)} .
\end{aligned}
$$

For $0<q \leq 1$ and $d \geq 0$ the above numerator polynomial is always greater than zero. Hence condition (iii), the variability condition is satisfied.

Thus we may deduce

Theorem 3 The distribution of the number of ascents in samples of $n$ geometric random variables converges to a Gaussian distribution with a speed of convergence of $O\left(n^{-1 / 2}\right)$, where the mean $\mu_{n}$ and the variance $\sigma_{n}^{2}$ are as given in Theorems 1 and 2 .

Remark In Flajolet and Sedgewick [4] it is also shown that under the conditions of the proposition, the mean $\mu_{n}$ and variance $\sigma_{n}^{2}$ are of the form

$$
\mu_{n}=\mathfrak{m}\left(\frac{\rho(1)}{\rho(u)}\right) n+O(1), \quad \sigma_{n}^{2}=\mathfrak{v}\left(\frac{\rho(1)}{\rho(u)}\right) n+O(1),
$$

where

$$
\mathfrak{m}(f)=\frac{f^{\prime}(1)}{f(1)} \quad \text { and } \quad \mathfrak{v}(f)=\frac{f^{\prime \prime}(1)}{f(1)}+\frac{f^{\prime}(1)}{f(1)}-\left(\frac{f^{\prime}(1)}{f(1)}\right)^{2}
$$


This gives

$$
\mu_{n}=\left.\left(\frac{1}{\rho(u)}\right)^{\prime}\right|_{u=1} n+O(1)=\frac{q^{d}}{1+q} n+O(1)
$$

which is in agreement with our exact result in Theorem 1.

In the case of the variance we must compute

$$
\begin{aligned}
\left.\mathfrak{v}\left(\frac{\rho(1)}{\rho(u)}\right)\right|_{u=1} & =\left.\left(\frac{1}{\rho(u)}\right)^{\prime \prime}\right|_{u=1}+\frac{q^{d}}{1+q}-\frac{q^{2 d}}{(1+q)^{2}} \\
& =\left.\left(-\rho^{\prime \prime}(u)+2 \rho^{\prime}(u)\right)\right|_{u=1}+\frac{q^{d}}{1+q}-\frac{q^{2 d}}{(1+q)^{2}} \\
& =\frac{2 q^{3 d}(1-q)}{(1+q)\left(1-q^{3}\right)}-\frac{3 q^{2 d}}{(1+q)^{2}}+\frac{q^{d}}{1+q} .
\end{aligned}
$$

So the variance is of the form

$$
\sigma_{n}^{2}=\left[\frac{2 q^{3 d}(1-q)}{(1+q)\left(1-q^{3}\right)}-\frac{3 q^{2 d}}{(1+q)^{2}}+\frac{q^{d}}{1+q}\right] n+O(1) .
$$

which (fortunately!) again corresponds to the main term of the exact result found earlier in Theorem 2.

\section{References}

[1] G. E. Andrews, R. Askey, and R. Roy. Special Functions. Cambridge University Press, 1999.

[2] P. Billingsley. Probability and Measure. John Wiley and Sons, 2nd edition, 1986.

[3] P. Flajolet and H. Prodinger. Level number sequences for trees. Discrete mathematics, 65:149-156, 1997.

[4] P. Flajolet and R. Sedgewick. Analytic Combinatorics, Symbolic Combinatorics (Chapters 1-9). http://algo.inria.fr/flajolet/Publications/books.html, November 2004.

[5] A. Knopfmacher and H. Prodinger. On Carlitz compositions. European Journal of Combinatorics, 19:579-589, 1998.

[6] A. Knopfmacher and H. Prodinger. The number of descents in samples of geometric random variables, Trees, Combinatorics and Probabilities. Birkhäuser Verlag, Trends in Mathematics, September 2004. 
